\title{
The association between multimorbidity and hospitalization is modified by individual demographics and physician continuity of care: a retrospective cohort study
}

Andrea Gruneir ${ }^{1,2^{*}}$, Susan E. Bronskill ${ }^{2,3}$, Colleen J. Maxwell ${ }^{2,4}$, Yu Qing Bai ${ }^{3}$, Anna J. Kone ${ }^{5}$, Kednapa Thavorn ${ }^{6}$, Yelena Petrosyan ${ }^{3}$, Andrew Calzavara ${ }^{2}$ and Walter P. Wodchis ${ }^{2,4}$

\begin{abstract}
Background: Multimorbidity poses a significant clinical challenge and has been linked to greater health services use, including hospitalization; however, we have little knowledge about the influence of contextual factors on outcomes in this population. Objectives: To describe the extent to which the association between multimorbidity and hospitalization is modified by age, gender, primary care practice model, or continuity of care (COC) among adults with at least one chronic condition.

Methods: A retrospective cohort study with linked population-based administrative data. Setting: Ontario, Canada. Cohort: All individuals 18 and older with at least one of 16 priority chronic conditions as of April 1, 2009 (baseline). Main Outcome Measures: Any hospitalization, 3 or more hospitalizations, non-medical discharge delay, and 30-day readmission within the 1 year following baseline.

Results: Of 5,958,514 individuals, 484,872 (8.1\%) experienced 646,347 hospitalizations. There was a monotonic increase in the likelihood of hospitalization and related outcomes with increasing multimorbidity which was modified by age, gender, and COC but not primary care practice model. The effect of increasing multimorbidity was greater in younger adults than older adults and in those with lower $\mathrm{COC}$ than with higher $\mathrm{COC}$. The effect of increasing multimorbidity on hospitalization was greater in men than women but reversed for the other outcomes.
\end{abstract}

Conclusions: The effect of multimorbidity on hospitalization is influenced by age and gender, important considerations in the development of person-centred care models. Greater continuity of physician care lessened the effect of multimorbidity on hospitalization, further demonstrating the need for care continuity across providers for people with chronic conditions.

Keywords: Chronic conditions, Age, Sex and gender, Continuity of care, Readmission

\footnotetext{
* Correspondence: gruneir@ualberta.ca

${ }^{1}$ Department of Family Medicine, University of Alberta, 6-40 University

Terrace, Edmonton, Alberta T6G 2T4, Canada

${ }^{2}$ Institute for Clinical Evaluative Sciences, 2075 Bayview Avenue, G-Wing,

Toronto, Ontario M4N 3M5, Canada

Full list of author information is available at the end of the article
} 


\section{Background}

Multimorbidity, the co-occurrence of two or more chronic conditions, is an important issue in clinical care and population health [1, 2]. Several studies have demonstrated strong associations between the burden of multimorbidity and health services use, especially hospitalizations [3-6]. Addressing the needs of individuals with multimorbidity continues to be a vexing clinical challenge due in part to the lack of appropriate evidence-based guidelines [1]. Patient care is also challenged by health systems that are organized around health care providers focused on single conditions with payment systems that reinforce that model [7]. This mismatch between population needs and health system design has prompted calls for person-centred care practices with a renewed focus on primary care [8-10].

Important gaps persist in our understanding of how patient and health system context influence service utilization and outcomes for people with multimorbidity. Context encompasses the range of biological, social, environmental, and health system factors that impact on health but that are often ignored or "adjusted" in research on multimorbidity [11]. A prime example is the treatment of age and gender - two fundamental individual characteristics that are associated with multimorbidity and health care use $[5,12]$ but have received little attention beyond treatment as confounding variables.

There is also little evidence regarding the role of health system factors, a significant omission given the potential for alternate health care provider arrangements, including physician payments and access to interdisciplinary resources, to facilitate patient-centred care. Moreover, specialists play an important role in the care of individuals with chronic conditions, and their use increases with the number of chronic conditions [13] This has implications for continuity of care, which has been shown to improve outcomes; although the same has yet to be investigated in the context of multimorbidity where involvement of a greater number of specialists can be expected.

The purpose of this study is to explore the role of key individual demographic and health system contextual factors in influencing the association between multimorbidity and hospitalization. Our objectives are to describe hospitalizations and related outcomes among a population-based cohort of adults by their degree of multimorbidity, and to test whether age, gender, primary care practice model, or continuity of care modify the association between multimorbidity and hospitalization. This research should help to target strategies to high-risk groups and suggest system responses to mitigate the impact of multimorbidity. We also aim to demonstrate the importance of incorporating context into the study of multimorbidity, an issue that has been highlighted by others as critical to moving the field forward [11].

\section{Methods}

\section{Setting}

This study was conducted in Ontario, Canada's largest province with approximately 13 million residents. The provincial health insurance program insures virtually all residents for physician and hospital care.

\section{Study design and data}

This is a retrospective cohort study conducted using linked population-based administrative data. These data include: the Registered Persons Database for demographic information on all residents with provincial health insurance; the Discharge Abstract Database (DAD) for data from all hospital discharges; the Ontario Health Insurance Plan (OHIP) claims database for physician billing claims; and the Client Agency Program Enrolment database for patient enrolment to primary care physicians and the practice model type. These data are regularly used in research [14-16].

The data were linked using unique encoded identifiers and are maintained at the Institute for Clinical Evaluative Sciences (ICES), where the data were analyzed. This study was approved by the Research Ethics Board at Sunnybrook Health Sciences Centre.

We included all Ontario residents over the age of 18 years with one of 16 chronic conditions as of April 1, 2009 (baseline): acute myocardial infarction, asthma, cancer, cardiac arrhythmia, chronic obstructive pulmonary disease, congestive heart failure, coronary syndrome, dementia, diabetes, hypertension, mood disorders, osteoarthritis, osteoporosis, renal failure, rheumatoid arthritis, or stroke. We selected these conditions based on prevalence and system burden [17-20]. Each condition was defined as the presence of two physician billing codes within 2 years or one hospital diagnostic code anytime between April 1, 2001 and baseline (or between April 1, 2007 and baseline for cancer or mood disorders) (See Appendix). We categorized the cohort as having one through five or more conditions at baseline. We used a simple count since there are no existing methods for creating meaningful clusters, especially without a central index condition. We included those with a single condition, even though this is not considered multimorbidity, to characterize the increasing risk of hospitalization with additional chronic conditions.

\section{Hospitalizations}

We followed each cohort member from baseline for up to 1 year and captured all unplanned hospital episodes. We examined four measures of hospital use within the year: 1) any hospitalization; 2) three or more hospitalizations; 3) hospitalizations with non-medical discharge delay; and 4) 30-day hospital readmission following discharge. For each hospital episode, we characterized the most responsible diagnosis for the admission, the length 
of stay, and discharge due to death. We also determined if the most responsible diagnosis for the admission matched a pre-existing condition recorded at baseline, an incident condition, or another reason. Non-medical discharge delays were identified using "alternate level of care" days which refer to periods when a patient no longer requires hospital services but cannot be discharged due to inadequate services elsewhere (for example, when a long-term care bed is required but not available) [21]. Hospital readmissions were counted only among those discharged alive.

\section{Contextual factors}

The contextual factors were: age, gender, primary care practice model, and continuity of care. The cohort was stratified as 18-64 years and 65-105 years. We chose this simple age stratification since this is a preliminary exploration on the differences between younger and older adults.

The broad distinguishing characteristics of the primary care practice models in Ontario are the degree of cooperation across physicians, the integration of other providers, and reimbursement type, which lead to different incentives and care practices [22]. Based on funding and interdisciplinary access, we created a three-level variable to describe the primary care practice model: a) non-capitated includes all non-rostered models where physicians largely operate on a fee-for-service basis; b) capitated includes models funded primarily via an age-sex adjusted capitation scheme; and c) capitated + includes models which operate similar funding to the capitated group but with additional payments for interdisciplinary care [23]. We anticipated that the association between multimorbidity and hospitalization would be weaker among individuals who belong to capitated and capitated + primary care practice models because of the greater emphasis on chronic condition management.

Continuity of care was measured using the Bice Concentration of Care Index (COC), which is an expression of the dispersion of physician visits over time across the number of individual physicians visited [24]. The index has a maximum score of 1.0 if all visits are to one physician and approaches zero with additional physicians visited; it accounts for the increase in the number of visits associated with an increasing number of physicians involved in care. We calculated the COC using billing codes for any outpatient physician visit, including all primary and specialty care visits, over the year prior to baseline. We included both primary care and specialist visits since individuals with chronic conditions may receive a significant share of their care from specialists [25] and since specialists are often involved in the management of common conditions [13]. Using the cohort median COC score (0.52), we categorized the cohort as either having a high concentration of care (greater continuity) or a low concentration (less continuity) and anticipated that higher $\mathrm{COC}$ would be protective against hospitalization and related outcomes.

\section{Analyses}

We described the frequency of hospitalization by number of chronic conditions. We used logistic regression to estimate the association between the degree of multimorbidity and each of: any hospitalization, three or more hospitalizations, non-medical discharge delay, and hospital readmission within 30 days of discharge during the follow-up. The likelihood of any hospitalization was modeled for the entire cohort but the likelihood of three or more hospitalizations and non-medical discharge delay was modeled only among those with at least one hospitalization. The likelihood of 30-day readmission was modeled only among those discharged alive from a prior hospitalization.

To assess for effect measure modification, we created a set of mutually exclusive variables to cross-classify individuals by degree of multimorbidity and each contextual factor. For example, we classified each individual as: age $<65$ years with one condition, age $<65$ years with two conditions, etc. We created a total of 16 logistic regression models (one per contextual factor per outcome). This strategy allows for direct estimation of the effect of multimorbidity on outcomes at each level of the contextual factor [26] so that we could directly compare odds ratios within and between levels of the contextual factor. The reference categories were set as those anticipated to have the lowest risk for hospitalization: younger age (1864 years), female, capitated + primary care practice model, and higher COC. The models testing age and gender were unadjusted for other variables but the models testing primary care practice model and continuity of care were adjusted for age and gender.

All analyses were completed using SAS version 9.3.

\section{Results}

We identified 5,958,514 individuals with at least one of 16 conditions. Nearly $52 \%$ of individuals had two or more conditions and $5.8 \%$ had five or more (Table 1). Mean age for the cohort was 53.0 (standard deviation $=18.0$ ) years but this varied from $44.9(\mathrm{SD}=15.8)$ years among those with one condition to $73.6(\mathrm{SD}=11.9)$ among those with five or more. Fifty-five percent of the cohort was female. The most prevalent priority conditions were osteoarthritis (44.4\%), hypertension (43.4 \%), and mood disorders $(22.7 \%)$. The majority were enrolled in a non-capitated primary care practice model $(66.7 \%)$. Continuity of care was relatively stable by degree of multimorbidity.

In the year following baseline, 484,872 individuals (8.1\%) were hospitalized 646,347 times. The frequency of hospitalization and related outcomes increased markedly 
Table 1 Baseline characteristics of adults, aged 18 years and older, with at least one priority chronic condition, by burden of multimorbidity (Ontario, Canada, April 1, 2009)

\begin{tabular}{|c|c|c|c|c|c|c|}
\hline & \multicolumn{5}{|c|}{ Number of priority chronic conditions at baseline } & \multirow[b]{2}{*}{ Total } \\
\hline & 1 & 2 & 3 & 4 & $5+$ & \\
\hline Number of Individuals ${ }^{a}$ & $2844033(47.7)$ & $1548197(26.0)$ & $812429(13.6)$ & $405766(6.8)$ & $348089(5.8)$ & $5958514(100)$ \\
\hline Age (in years), mean (SD) & $44.9(15.8)$ & $54.5(16.2)$ & $62.4(14.9)$ & $69.0(13.5)$ & $73.6(11.9)$ & $53.0(18.0)$ \\
\hline \multicolumn{7}{|l|}{ Age Groups, N (\%) } \\
\hline$<65$ years & $2523875(88.7)$ & $1124098(72.6)$ & $440940(54.3)$ & $157162(38.7)$ & $78049(22.4)$ & $4324124(72.6)$ \\
\hline$\geq 65$ years & $320158(11.3)$ & $424099(27.4)$ & $371489(45.7)$ & $248604(61.3)$ & $270040(77.6)$ & $1634390(27.4)$ \\
\hline Female, N (\%) & $1477561(52.0)$ & $872671(56.4)$ & $472030(58.1)$ & $234217(57.7)$ & $194732(55.9)$ & $3251211(54.6)$ \\
\hline \multicolumn{7}{|l|}{ Prevalence of Each Priority Condition, N (\%) } \\
\hline Osteoarthritis & 785297 (27.6) & $777387(50.2)$ & $523568(64.4)$ & $289806(71.4)$ & $269393(77.4)$ & $2645451(44.4)$ \\
\hline Rheumatoid arthritis & $11447(0.4)$ & $30963(2.0)$ & $34028(4.2)$ & $25879(6.4)$ & $32116(9.3)$ & $134433(2.3)$ \\
\hline Cancer & $197688(7.0)$ & $226464(14.6)$ & $181622(22.4)$ & $116682(28.8)$ & $122160(35.1)$ & $844616(14.2)$ \\
\hline Arrhythmia & $26284(0.9)$ & $44264(2.7)$ & $57281(7.1)$ & $59136(14.6)$ & 116922 (33.6) & $303887(5.1)$ \\
\hline Dementia & $8695(0.3)$ & $20556(1.3)$ & $29767(3.7)$ & $30878(7.6)$ & $58159(16.7)$ & $148055(2.5)$ \\
\hline Mood disorders & $470962(16.6)$ & $401028(25.9)$ & $233008(28.7)$ & $125289(30.9)$ & $119660(34.4)$ & $1349947(22.7)$ \\
\hline Osteoporosis & $41424(1.5)$ & $72486(4.7)$ & $70425(8.7)$ & $48836(12.0)$ & $52837(15.2)$ & $286008(4.8)$ \\
\hline Renal disease & $5581(0.2)$ & $16568(1.1)$ & $27301(3.4)$ & $29958(7.4)$ & $68164(19.6)$ & $147572(2.5)$ \\
\hline Stroke & $5549(0.2)$ & $15923(1.0)$ & $25059(3.1)$ & $26025(6.4)$ & $50319(14.5)$ & $122875(2.1)$ \\
\hline Coronary artery disease & $33552(1.2)$ & $99472(6.4)$ & $147047(18.1)$ & $139099(34.3)$ & $205730(59.1)$ & $624900(10.5)$ \\
\hline Asthma & $515746(18.1)$ & 323609 (20.9) & $190172(23.4)$ & $112921(27.8)$ & 130775 (37.6) & $1273223(21.4)$ \\
\hline Congestive heart failure & $2309(0.1)$ & $10806(0.7)$ & $27568(3.4)$ & $44045(10.9)$ & $131444(37.8)$ & $216172(3.6)$ \\
\hline Chronic obstructive pulmonary disease & $13215(0.5)$ & $33266(2.2)$ & $49300(6.1)$ & $52272(12.9)$ & $108959(31.3)$ & $257012(4.3)$ \\
\hline Hypertension & $573702(20.2)$ & $756405(48.7)$ & $585695(72.1)$ & $346365(85.4)$ & $326183(93.7)$ & $2588350(43.4)$ \\
\hline Diabetes mellitus & $152497(5.4)$ & $266126(17.2)$ & $253328(31.2)$ & $173209(42.7)$ & $190688(54.8)$ & $1035848(17.4)$ \\
\hline Acute myocardial infarction & $85(0.0)$ & $1071(0.1)$ & $2118(0.3)$ & $2664(0.7)$ & $7093(2.0)$ & $13031(0.2)$ \\
\hline \multicolumn{7}{|l|}{ Primary Care Practice Model, ${ }^{\mathrm{b}}$ N (\%) } \\
\hline Non-capitated & $1921317(67.6)$ & $1020886(65.9)$ & $535298(65.9)$ & $267574(65.9)$ & $231673(66.6)$ & $3976748(66.7)$ \\
\hline Capitated & $442850(15.6)$ & $258985(16.7)$ & $137301(16.9)$ & $68569(16.9)$ & $58198(16.2)$ & $965903(16.2)$ \\
\hline Capitated+ & $479866(16.9)$ & $268326(17.3)$ & $139830(17.2)$ & $69623(17.2)$ & $58218(16.7)$ & $1015863(17.1)$ \\
\hline \multicolumn{7}{|l|}{ Continuity of Care ${ }^{c}$} \\
\hline Mean (SD) & $0.6(0.3)$ & $0.7(0.3)$ & $0.7(0.3)$ & $0.7(0.3)$ & $0.6(0.3)$ & $0.6(0.3)$ \\
\hline Median (Q1-Q3) & $0.5(0.3-0.8)$ & $0.5(0.3-0.8)$ & $0.5(0.3-0.8)$ & $0.5(0.3-0.8)$ & $0.5(0.3-0.7)$ & $0.5(0.3-0.8)$ \\
\hline High (>0.52), N (\%) & $1244438(43.8)$ & 802231 (51.8) & $430849(53.0)$ & $203662(50.2)$ & $147651(42.4)$ & $2828831(47.5)$ \\
\hline Low $(<=0.52), \mathrm{N}(\%)$ & 1599595 (56.2) & 745966 (48.2) & $381580(47.0)$ & 202104 (49.8) & 200438 (57.6) & $3129683(52.5)$ \\
\hline
\end{tabular}

aparentheses show row percent

${ }^{b}$ Primary care practice models defined as: non-capitated models include non-rostered models and those that operate on a fee-for-service basis; capitated models include family health networks and family health organizations operating on a age-sex adjusted capitation funding scheme; and the capitated + models include family health teams and other rostered models operating on a capitated funding scheme with additional incentives for interdisciplinary care

c Calculated using the Bice Concentration of Care Index

with multimorbidity (Table 2). The proportion with at least one hospitalization increased from $4.6 \%$ among those with one condition to $26.9 \%$ among those with five or more conditions. Among those hospitalized, $4.3 \%$ with one condition experienced three or more hospitalizations compared to $13.8 \%$ with five or more conditions. Hospitalizations with non-medical discharge delay, inpatient death, and 30-day readmission also increased with multimorbidity.
The direct, unadjusted effect of multimorbidity on outcomes is shown in Table 3. In all cases, there is a clear monotonic increase in the ORs with tight $95 \%$ CIs.

Figure 1a-d illustrates the ORs and $95 \%$ CIs generated from cross-classifying multimorbidity with each contextual factor. To assess for effect measure modification, we compared the odds ratio of the five or more condition group against the odds ratio for the single condition group 
Table 2 Characteristics of all hospitalizations within 1 year for adults, aged 18 years and older with at least one priority chronic condition, Ontario, Canada, April 1, 2009 - March 31, 2010

\begin{tabular}{|c|c|c|c|c|c|c|}
\hline & \multicolumn{5}{|c|}{ Number of priority chronic conditions } & \multirow[b]{2}{*}{ Total } \\
\hline & 1 & 2 & 3 & 4 & $5+$ & \\
\hline Number of Individuals & 2844033 & 1548197 & 812429 & 405766 & 348089 & 5958514 \\
\hline Any hospitalization, N (\%) & $131029(4.6)$ & $108939(7.0)$ & $87273(10.7)$ & $64120(15.8)$ & $93511(26.9)$ & $484872(8.1)$ \\
\hline $3+$ hospitalizations, $\mathrm{N}\left(\%^{\mathrm{a}}\right)$ & $5581(4.3)$ & $6680(6.1)$ & $6893(7.9)$ & $6048(9.4)$ & $12907(13.8)$ & $38109(7.9)$ \\
\hline \multicolumn{7}{|l|}{ Hospitalizations } \\
\hline Total Number of Hospitalizations & 156507 & 138190 & 116481 & 89293 & 145876 & 646347 \\
\hline Hospitalization for an existing chronic condition, N (\% $\left.{ }^{b}\right)$ & $10,963(7.0)$ & $20,304(14.7)$ & $22,392(19.2)$ & $19,305(21.6)$ & $35,224(24.1)$ & $108,188(16.7)$ \\
\hline Hospitalization for a new chronic condition, N (\% $\left.{ }^{\mathrm{b}}\right)$ & $14503(9.3)$ & $14700(10.6)$ & $12381(10.6)$ & $8949(10.0)$ & $14401(9.9)$ & $64934(10.0)$ \\
\hline Length of stay, days Median (IQR) & $3(1-5)$ & $3(2-6)$ & $4(2-8)$ & $5(2-9)$ & $6(3-11)$ & $4(2-7)$ \\
\hline Non-medical discharge delay, $\mathrm{N}\left(\%^{\mathrm{b}}\right)$ & $4832(3.1)$ & $7769(5.6)$ & $9477(8.1)$ & $8901(10.0)$ & $17622(12.1)$ & $48601(7.5)$ \\
\hline Discharge due to death, $N\left(\%^{b}\right)$ & $3942(3.0)$ & $6138(5.6)$ & $7041(8.1)$ & $6667(10.4)$ & $14111(15.1)$ & 37899 (7.8) \\
\hline 30-day readmission among those discharged alive, $\mathrm{N}\left(\%^{\mathrm{C}}\right)$ & $9284(7.3)$ & $10076(9.8)$ & $9503(11.8)$ & 8171 (14.2) & $15842(20.0)$ & $52876(11.8)$ \\
\hline
\end{tabular}

IQR interquartile range

${ }^{a} \%$ of individuals with any hospitalization; ${ }^{\mathrm{b}} \%$ of all hospitalizations; ${ }^{{ }^{c}} \%$ of all hospitalizations where patient was discharged alive

within each level of the contextual factor (for example, within younger adults) and then compared that ratio across levels of the contextual factor (between younger and older adults). Approximately $5.6 \%$ of those under 65 were hospitalized at least once relative to $14.8 \%$ of those over 65 years (not shown). Age appeared to modify the association between multimorbidity and hospitalization (Fig. 1a). In younger adults, the odds of hospitalization increased 5.4-fold from one condition to five or more conditions, whereas for older adults, the odds increased 4.8-fold with multimorbidity (younger adults: 5.4/1.0 vs. older adults: 9.2/1.9). Similar patterns emerged for the other outcomes. See Additional file 1 TableS1-S4 for the number and proportion of individuals who experienced each outcome by age, sex, primary care practice model and continuity of care.

Among those with a single condition, men had $40 \%$ lower odds of hospitalization than women; however, the association reversed with multimorbidity (Fig. 1b). Among women, the odds of hospitalization increased five-fold with degree of multimorbidity; among men the same odds increased nearly 11-fold with degree of multimorbidity (women: 5.5/1.0 vs men: 6.5/0.6). Men showed greater odds of each three or more hospitalizations, non-medical discharge delay, and 30-day readmission among those with a single condition, but the relative increase in the odds with multimorbidity was consistently greater for women on all outcomes (even though the odds of three or more hospitalizations and 30-day readmission were higher for men at each level of multimorbidity).

After adjusting for age and gender, individuals in capitated and non-capitated primary care practice models showed lower odds of hospitalization, three or more hospitalizations, and 30-day readmissions compared to individuals in capitated + practice models. There was no difference in the relative odds of hospitalization or related outcomes with increasing multimorbidity across primary care practice models indicating no effect measure modification (Fig. 1c).

The modifying effect of $\mathrm{COC}$ was inconsistent across outcomes. Among those with low continuity $(\mathrm{COC} \leq 0.52)$, the odds of hospitalization increased over five-fold with greater multimorbidity but only four-fold in those with high continuity (low continuity: 7.0/1.3 vs. high continuity: 4.1/1.0). A similar pattern emerged for 30-day readmissions but no such differences were observed for the other outcomes (Fig. 1d).

\section{Discussion}

In this population-based study of nearly six million adults with at least one of 16 chronic conditions, we

Table 3 Direct effect of multimorbidity on the odds of hospitalization and related outcomes within 1 year among adults, aged 18 years and older with at least one priority chronic condition, by burden of multimorbidity, April 1, 2009 - March 31, 2010

\begin{tabular}{|c|c|c|c|c|c|}
\hline & \multicolumn{5}{|c|}{ Number of chronic conditions } \\
\hline & 1 & 2 & 3 & 4 & $5+$ \\
\hline Any hospitalization, OR (95\% Cl) & Ref & $1.6(1.5-1.6)$ & $2.5(2.5-2.5)$ & $3.9(3.8-3.9)$ & $7.6(7.5-7.6)$ \\
\hline Three or more hospitalizations among those hospitalized, OR (95 \% Cl) & Ref & $1.6(1.5-1.6)$ & $2.1(2.1-2.2)$ & $2.7(2.7-2.8)$ & $4.3(4.2-4.4)$ \\
\hline Any days with non-medical discharge delay among those hospitalized, OR (95 \% Cl) & Ref & $2.0(2.0-2.1)$ & $3.2(3.1-3.3)$ & $4.2(4.1-4.4)$ & $6.0(5.9-6.2)$ \\
\hline 30-day readmission among those discharged alive, OR (95\% Cl) & Ref & $1.4(1.4-1.4)$ & $1.7(1.7-1.8)$ & $2.1(2.0-2.2)$ & $3.1(3.1-3.2)$ \\
\hline
\end{tabular}




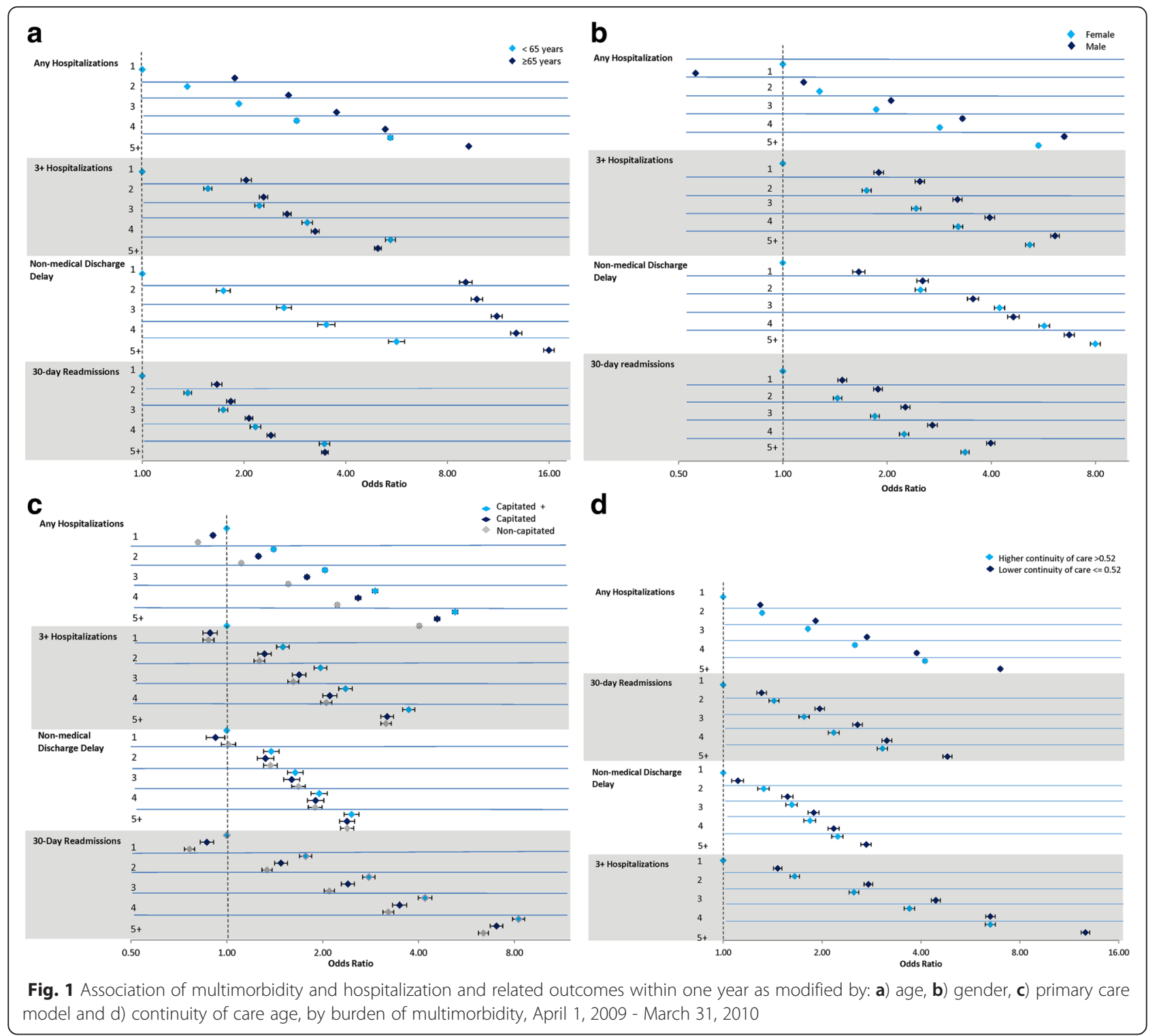

found a high prevalence of multimorbidity that was strongly associated with hospitalization. Our findings suggest that hospitalizations also become more complicated with increasing multimorbidity as demonstrated by longer stays, increased frequency of non-medical discharge delays and in-hospital death, as well as, a greater likelihood of multiple hospitalizations, including 30-day readmissions. These findings reinforce calls for better integration of patient-centred care practices both within and across health care sectors and the sharing of information to support care for those with multimorbidity.

We found that the association between multimorbidity and hospitalization was modified by both age and gender, two important contextual factors that are often overlooked. The prevalence of multimorbidity increases with age and much of the literature focuses on older adults, highlighting its importance in older populations, [5] but in our study, as in others, there were more younger adults than older adults living with multimorbidity [27, 28]. Further, the impact of increasing multimorbidity was relatively greater in younger adults than in older adults even though older adults were consistently at greater risk of hospitalization and related outcomes. The reason for the differential effect is unknown but may result from different clustering of chronic conditions by age and/ or a degree of vulnerability inherent to having multiple chronic conditions at a young age that results in worse relative symptoms and outcomes.

The impact of gender on the association between multimorbidity and hospitalization was not consistent across outcomes. Men were more likely to be affected by 
multimorbidity when it came to hospitalization but women were more likely to be affected by multimorbidity on all other outcomes. This is likely attributed to underlying social differences, such as income, living arrangements, and access to informal caregivers, which tend to put women, especially older women, at greater risk for requiring supportive care services [29]. While some research shows that women experience a higher burden of multimorbidity, this has not been consistent $[12,30]$ and it is not yet clear how this relates to health services utilization given that men and women also have different patterns of health seeking behaviour.

A recent publication details the contextual factors identified by stakeholders as critical to moving the research agenda on multimorbidity forward [11]. Neither age nor gender were explicitly identified among that long list even though both cut across their broad categories of biological, person, and family factors. Our results demonstrate the importance of considering the moderating impact of age and gender and the influence of different biological, health behaviour, and social structures on health outcomes among people with multimorbidity. Future research should also consider the intersection of these factors as well as others, such as ethnicity and socioeconomic standing [31].

With respect to health system factors, the results were mixed. Attachment to a capitated + primary care practice model was associated with poorer outcomes than other models and there was no evidence of effect modification. It is difficult to know why this pattern emerged. Some research suggests that practice models such as those in the capitation + group have a higher case-mix of chronic conditions and multimorbidity than other practices, [32] meaning that preventing hospitalization may be more challenging among their patients. One Ontario-based evaluation found more emergency department visits among patients in capitated models but suggested that this was due to patterns of use that pre-dated funding changes [22]. Alternatively, and perhaps most importantly, primary care practice model may be a poor proxy for quality of care and future research should focus on specific aspects of service delivery.

Continuity of care, measured across all the physicians visited over 1 year, did modify the association between multimorbidity and hospitalization. The effect of multimorbidity on hospitalization, multiple hospitalizations, and 30-day readmissions was less pronounced among individuals with greater continuity than those with lesser continuity. The same was not observed for non-medical discharge delays, likely because of different health system drivers. The Bice Index is a proxy for relationship continuity based solely on the dispersion of visits across physicians and does not account for other aspects such as informational continuity [33]. Regardless, we demonstrate that when visits are concentrated with a single physician, even among individuals with multimorbidity, the risk of hospitalization is reduced. The magnitude was particularly remarkable as individuals with less than median $\mathrm{COC}$ experienced outcomes that were equivalent to those with high continuity with one less chronic condition. The benefits of having one provider who can provide oversight for patients with complex needs has been demonstrated by others $[34,35]$ and has been reflected in upcoming changes to the U.S. Medicare fee schedule [7].

This study has limitations. First, we focused on 16 conditions, likely underestimating the burden of multimorbidity; however, these are highly prevalent conditions and are consistent with those used to report on multimorbidity elsewhere [6]. Second, there is the potential for diagnostic misclassification due to the administrative data. Although many of our diagnostic algorithms have high sensitivity and specificity, others do not. Third, we used a simple approach to operationalizing multimorbidity by strictly counting conditions and could not account for severity or symptom burden. Our diagnostic count does not address concordant, discordant, or other combinations used to examine comorbidity in specific diagnostic groups [36]. However, with no obvious method for clustering conditions, a simple diagnostic counts is the most straightforward method to define multimorbidity across populationbased studies such as ours.

\section{Conclusions}

We found that demographic and modifiable health system factors influenced the impact of multimorbidity on the risk of hospitalization and related outcomes. Our findings on age and gender help to identify groups that may be at particularly high risk for adverse outcomes associated with multimorbidity; however, additional research is required to better characterize this vulnerability including attention to other demographic factors and the mechanisms through which they influence outcomes. Our findings also support the importance of continuity of care for complex patients. Chronic condition management programs must incorporate levers to promote continuity, especially for patients who are likely to see multiple providers. While primary care is the obvious setting for coordinated and integrated care, our study was unable to determine differences across practice model types. Additional research on key aspects of chronic condition management in primary care and the impact on people with multimorbidity are still required.

\section{Availability of data and materials}

Data are available from the Institute for Clinical Evaluative Sciences for researchers who meet the criteria for access to confidential data. 


\section{Appendix}

Table 4 List of diagnosis codes for defining the 16 selected conditions

\begin{tabular}{|c|c|c|}
\hline Condition & ICD 9/OHIP & ICD 10 \\
\hline AMI & 410 & 121,122 \\
\hline Arthritis - Osteoarthritis & 715 & M15-M19 \\
\hline $\begin{array}{l}\text { Arthritis - Other Arthritis (Synovitis, Fibrositis, } \\
\text { Connective tissue disorders, Ankylosing spondylitis, } \\
\text { Gout Traumatic arthritis, pyogenic arthritis, Joint } \\
\text { derangement, Dupuytren's contracture, Other } \\
\text { MSK disorders) }\end{array}$ & $727,729,710,720,274,716,711,718,728,739$ & $\begin{array}{l}\text { M00-M03, M07, M10, M1 1-M14, M20-M25, } \\
\text { M30-M36, M65-M79 }\end{array}$ \\
\hline Arthritis - Rheumatoid arthritis & 714 & M05-M06 \\
\hline Asthma & 493 & $J 45$ \\
\hline Cancer & $140-239$ & C00-C26, C30-C44, C45-C97, \\
\hline Cardiac Arrythmia & $427.3(\mathrm{DAD}) / 427(\mathrm{OHIP})$ & $148.0,148.1$ \\
\hline $\mathrm{CHF}$ & 428 & $1500,1501,1509$ \\
\hline COPD & $491,492,496$ & $J 41, J 43, J 44$ \\
\hline Dementia & $\begin{array}{l}\text { 290, 331, } 797 \text { (OHIP)/290.0, 290.1, 290.3, 290.4, } \\
\text { 290.8, 290.9, 294.1, 294.8, 294.9, 331.0, 331.1, } \\
\text { 331.2, } 797 \text { (DAD) }\end{array}$ & $\begin{array}{l}\text { F000, F001, F002, F009, F010, F011, F012, } \\
\text { F013, F018, F019, F020, F021, F022, F023, } \\
\text { F024, F028, F03, F051, F065, F066, F068, } \\
\text { F069, F09, G300, G301, G308, G309, G310, } \\
\text { G311, R54 }\end{array}$ \\
\hline Depression & $311,300,296$ & F32, F33, F412, F480 \\
\hline Diabetes & 250 & E08 - E13 \\
\hline Hypertension & $401,402,403,404,405$ & $|10| 11,,|12| 13,, \mid 15$ \\
\hline Osteoporosis & 733 & M81 M82 \\
\hline Renal failure & $403,404,584,585,586, v 451$ & N17, N18, N19, T82.4, Z49.2, Z99.2 \\
\hline Stroke & $430,431,432,434,436$ & $160-164$ \\
\hline Coronary syndrome (excluding MI) & $411-414$ & $120,122-125$ \\
\hline
\end{tabular}

\section{Additional file}

Additional file 1: Supplementary Tables. Table S1. Number and proportion of individuals who experienced each outcome, stratified by age and number of priority chronic conditions (column percent shown). Table S2. Number and proportion of individuals who experienced each outcome, stratified by gender and number of priority chronic conditions (column percent shown). Table S3. Number and proportion of individuals who experienced each outcome, stratified by primary care practice model and number of priority chronic conditions (column percent shown). Table S4. Number and proportion of individuals who experienced each outcome, stratified by continuity of care and number of priority chronic conditions (column percent shown). (DOCX 147 kb)

\section{Abbreviations}

COC: continuity of care; ICES: Institute for Clinical Evaluative Sciences; IQR: interquartile range.

\section{Competing interests}

The authors declare that they have no competing interests.

\section{Authors' contributions}

AG conception, design, data access, data interpretation, first draft and all critical revisions of the manuscript. SB conception, design, data interpretation, all critical revisions of the manuscript. CM conception, design, data interpretation, all critical revisions of the manuscript. YB design, data analysis, interpretation, revisions to manuscript/tables/figures. AK conception, design, revisions to the manuscript/tables/figures. KT conception, design, revisions to the manuscript/tables/figures. YP conception, design, revisions to the manuscript/tables/figures. AC design, data analysis, interpretation, revisions to manuscript/tables/figures. WW conception, funding, design, data access, data interpretation, all critical revisions of the manuscript. All authors read and approved the final manuscript.

\section{Acknowledgments}

The project was funded by the Ontario Ministry of Health and Long-Term Care (MOHLTC), Health System Performance Research Network Grant. At the time this research was conducted, Dr. Gruneir was partially supported by Team Grant (OTG-88591) from the Canadian Institutes for Health Research $(\mathrm{ClHR})$ Institute of Nutrition, Metabolism, and Diabetes and Dr. Bronskill was supported by a CIHR New Investigator Award. Dr. Gruneir is currently supported by a ClHR New Investigator Award. This study was supported by the Institute for Clinical Evaluative Sciences (ICES), which is funded by an annual grant from the MOHLTC. The opinions, results and conclusions reported in this paper are those of the authors and are independent from the funding sources. No endorsement by ICES or the Ontario MOHLTC is intended or should be inferred. Parts of this material are based on data and information compiled and provided by the Canadian Institutes of Health Information (CIHI). However, the analyses, conclusions, opinions and statement expressed herein are those of the authors, and not necessarily those of $\mathrm{ClHI}$. The authors would like to acknowledge Dilzayn Panjwani, MSc at Women's College Hospital, Toronto, Ontario for her assistance in preparing the manuscript.

\section{Author details}

'Department of Family Medicine, University of Alberta, 6-40 University Terrace, Edmonton, Alberta T6G 2T4, Canada. ${ }^{2}$ Institute for Clinical Evaluative 
Sciences, 2075 Bayview Avenue, G-Wing, Toronto, Ontario M4N 3M5, Canada. ${ }^{3}$ Institute of Health Policy Management \& Evaluation, University of Toronto, 155 College Street, 4th Floor, Toronto, Ontario M5T 3M6, Canada. ${ }^{4}$ School of Pharmacy, University of Waterloo, 200 University Avenue West, Waterloo, Ontario N2L 3G1, Canada. ${ }^{5}$ Cancer Care Ontario, 620 University Ave, Toronto, Ontario M5G 2L7, Canada. ${ }^{6}$ Ottawa Hospital Research Institute, The Ottawa Hospital, 501 Smyth Road, PO Box 201B, Ottawa, Ontario K1H 8L6, Canada.

\section{Received: 26 September 2015 Accepted: 21 April 2016}

Published online: 27 April 2016

\section{References}

1. Tinetti ME, Fried TR, Boyd CM. Designing health care for the most common chronic condition-multimorbidity. JAMA. 2012;307:2493-4.

2. Pefoyo AJ, Bronskill SE, Gruneir A, Calzavara A, Thavorn K, Petrosyan Y, et al. The increasing burden and complexity of multimorbidity. BMC Public Health. 2015;15:415.

3. Canadian Institute for Health Information. Seniors and the Health Care System: What is the Impact of Multiple Chronic Conditions. Toronto: Canadian Institute for Health Information; 2011.

4. Freund T, Kunz CU, Ose D, Peters-Klimm F. Patterns of multimorbidity in primary care patients at high risk of future hospitalization. Popul Health Manag. 2012;15:119-24.

5. Marengoni A, Fratiglioni L. Disease clusters in older adults: rationale and need for investigation. J Am Geriatr Soc. 2011;59:2395-6.

6. Lochner KA, Goodman RA, Posner S, Parekh A. Multiple chronic conditions among Medicare beneficiaries: state-level variations in prevalence, utilization, and cost, 2011. Medicare Medicaid Res Rev. 2013;3(3)

7. Parekh AK, Kronick R, Tavenner M. Optimizing health for persons with multiple chronic conditions. JAMA. 2014;312:1199-200.

8. Boyd C, Fortin M. Future of Multimorbidity Research: How Should Understanding of Multimorbidity Inform Health System Design? Public Health Rev. 2010;32:451-74

9. American Geriatrics Society Expert Panel on the Care of Older Adults with Multimorbidity. Patient-centered care for older adults with multiple chronic conditions: a stepwise approach from the American Geriatrics Society: American Geriatrics Society Expert Panel on the Care of Older Adults with Multimorbidity. J Am Geriatr Soc. 2012;60:1957-68.

10. Affleck A, Parks P, Drummond A, Rowe BH, Ovens HJ. Position Statement on Emergency Department Overcrowding: Canadian Association of Emergency Physicians; 2007

11. Bayliss EA, Bonds DE, Boyd CM, Davis MM, Finke B, Fox MH, et al. Understanding the context of health for persons with multiple chronic conditions: moving from what is the matter to what matters. Ann Fam Med. 2014;12:260-9

12. Verbrugge LM, Patrick DL. Seven chronic conditions: their impact on US adults' activity levels and use of medical services. Am J Public Health. 1995; 85:173-82.

13. Starfield B, Lemke KW, Herbert R, Pavlovich WD, Anderson G. Comorbidity and the use of primary care and specialist care in the elderly. Ann Fam Med. 2005;3:215-22.

14. Bronskill SE, Anderson GM, Sykora K, Wodchis WP, Gill S, Shulman Kl, et al. Neuroleptic drug therapy in older adults newly admitted to nursing homes: incidence, dose, and specialist contact. J Am Geriatr Soc. 2004;52:749-55.

15. Chan BTB, MJ Schull, and SE Schultz. Emergency Department Services in Ontario 1993- 2000. Toronto: Institute for Clinical Evaluative Sciences; 2001.

16. Canadian Institute for Health Information. Quality Assurance Processes Applied to the Discharge Abstract and Hospital Morbidity Databases. Ottawa: $\mathrm{ClH}$; 2008

17. Schultz SE, Rothwell DM, Chen Z, Tu K. Identifying cases of congestive heart failure from administrative data: a validation study using primary care patient records. Chronic Dis Inj Can. 2013;33:160-6.

18. Hux JE, Ivis F, Flintoft V, Bica A. Diabetes in Ontario: determination of prevalence and incidence using a validated administrative data algorithm. Diabetes Care. 2002;25:512-6.

19. Tu K, Campbell NR, Chen ZL, Cauch-Dudek KJ, McAlister FA. Accuracy of administrative databases in identifying patients with hypertension. Open Med. 2007;1:e18-26.

20. Statistics Canada. Table 051-0001 Estimates of population, by age group and sex for July 1, Canada, provinces and territories. Ottawa, ON: Statistics Canada; 2014
21. Canadian Institute for Health Information. Alternate Level of Care in Canada. Toronto, ON: Canadian Institute for Health Information; 2009.

22. Glazier RH, Klein-Geltink J, Kopp A, Sibley LM. Capitation and enhanced fee-for-service models for primary care reform: a population-based evaluation. CMAJ. 2009;180:E72-81.

23. Family Practice Models. Government of Ontario, 2013. http://www. healthforceontario.ca/en/Home/Physicians/Training_/_Practising_Outside_ Ontario/Physician_Roles/Family_Practice_Models. Accessed 15 Apr 2015.

24. Bice TW, Boxerman SB. A quantitative measure of continuity of care. Med Care. 1977;15:347-9.

25. Romaire MA, Haber SG, Wensky SG, McCall N. Primary care and specialty providers: an assessment of continuity of care, utilization, and expenditures. Med Care. 2014:52:1042-9.

26. Greenland S, Rothman KJ. Chapter 18: Concepts of Interaction. In: Rothman KJ, Greenland S, editors. Modern Epidemiology. 2nd ed. New York, NY: Lippincott-Raven; 1998. p. 329-42.

27. Rocca WA, Boyd CM, Grossardt BR, Bobo WV, Finney Rutten $L$, Roger VL, et al. Prevalence of multimorbidity in a geographically defined American population: patterns by age, sex, and race/ethnicity. Mayo Clin Proc. 2014:89:1336-49.

28. Violán C, Foguet-Boreu Q, Roso-Llorach A, Rodriguez-Blanco T, Pons-Vigués M, Pujol-Ribera E, et al. Burden of multimorbidity, socioeconomic status and use of health services across stages of life in urban areas: a cross-sectional study. BMC Public Health. 2014;14:530.

29. Gruneir A, Forrester J, Camacho X, Gill SS, Bronskill SE. Gender differences in home care clients and admission to long-term care in Ontario, Canada: a population-based retrospective cohort study. BMC Geriatr. 2013;13:48.

30. Agborsangaya CB, Lau D, Lahtinen M, Cooke T, Johnson JA. Multimorbidity prevalence and patterns across socioeconomic determinants: a crosssectional survey. BMC Public Health. 2012;12:201.

31. McLean G, Gunn J, Wyke S, Guthrie B, Watt GC, Blane DN, et al. The influence of socioeconomic deprivation on multimorbidity at different ages: a cross-sectional study. Br J Gen Pract. 2014:64:e440-7.

32. Glazier RH, Zagorski BM JR. Comparison of Primary Care Models in Ontario by Demographics, Case Mix and Emergency Department Use, 2008/09 to 2009/10. Toronto: Institute of Clinical Evaluative Sciences; 2012.

33. Haggerty JL, Reid RJ, Freeman GK, Starfield BH, Adair CE, McKendry R. Continuity of care: a multidisciplinary review. BMJ. 2003;327:1219-21.

34. Menec VH, Sirski M, Attawar D, Katz A. Does continuity of care with a family physician reduce hospitalizations among older adults? J Health Serv Res Policy. 2006;11:196-201.

35. Saultz JW, Lochner J. Interpersonal continuity of care and care outcomes: a critical review. Ann Fam Med. 2005;3:159-66.

36. Piette JD, Kerr EA. The impact of comorbid chronic conditions on diabetes care. Diabetes Care. 2006;29:725-31.

\section{Submit your next manuscript to BioMed Central and we will help you at every step:}

- We accept pre-submission inquiries

- Our selector tool helps you to find the most relevant journal

- We provide round the clock customer support

- Convenient online submission

- Thorough peer review

- Inclusion in PubMed and all major indexing services

- Maximum visibility for your research

Submit your manuscript at www.biomedcentral.com/submit
) Biomed Central 